\title{
Post-Vaccination Immunity in FMD
}

\author{
AI Samuilenko* and CA Grin \\ All-Russian research and technological Institute of biological industry, Russia
}

Received: 㘹 February 21, 2018; Published: 眥 March 01, 2018

*Corresponding author: AI Samuilenko, All-Russian research and technological Institute of biological industry, Russia

\section{Short Communication}

The general opinion of scientists from different countries is that the 21st century is the age of biotechnology, one of the directions of which is the creation of bio products. Bio preparations are used to increase efficiency in the production of food products of plant and animal origin; neutralizing environmental pollutants with the production of useful products from wastes; ensuring the diagnosis, prevention and treatment of multi cellular organisms, including humans and solving many other problems of mankind. A special place is occupied by immunology, the science of the structure and functions of the immune system and the study of immunity, a specific biological property of multi cellular organisms, aimed at protecting against genetically alien factors (microorganisms, bacteria, viruses, plants and fungi), foreign molecules and others. Immunity also provides immunity to the body from infections when re-encountering the pathogen.

The modern theory of immunity is called clonal-selective: a clone of B-cells is formed and their selection is observed, that is, selection in the final analysis by antigen (with help of t-helpers). The authors of this theory were Nobel Prize winners F Bernet, N Erne, PB Medvar and other scientists. Immune protection is provided by two mechanisms: congenital and adaptive, which include cellular and humoral components and actively interact with each other during the immune response. The recognition of a multitude of alien antigens (1018) is due to the enormous diversity in our body of clones of T lymphocytes formed in the thymus and with the help of the genes of the main complex histo compatibility classes I and II. Neutralization of the foreign antigen is carried out by circulating antibodies in the body fluids (humoral immunity) and cytotoxic Lymphocytes (cellular immunity). The main characteristics of the immune response are the ability to distinguish your own antigens from foreign ones. Specificity and immune memory.

Regardless of the form of the immune response, it is traditionally divided into an inductive and an effective (productive) phase. In the inductive phase, the antigen is represented; the information about the antigen from the cells of innate immunity (antigen-presenting cells) is transferred to the initiators of adaptive immunity to T-helpers. Then the path of the further development of the immune response along the cellular or humoral path is chosen through induction of the differentiation of the T helper species (Th1, Th2, Th17, etc.). With the participation of these T-helpers there is a differentiation of effective immunocytes and parallel memory cells. An analysis of numerous publications showing that under the influence of mutagenic factors (chemical, physical and biological) on DNA, ultimately results in a change in its function. And under the influence of foreign antigen cells of the body produce a new specific production of antibodies. The purpose of this work is a comparative analysis of the results of the study of post vaccinal anti-melanoma immunity, taking into account the reproduction of the use of unique features of foot and mouth disease virus during its reproduction, in vivo, to cause the formation of sores in the tongue of cattle when administered under the mucous membrane and in vitro - when cultured in a reactor on cells the mucous membrane of the tongue of these animals. Studying immunity in animals vaccinated with vaccines containing different concentration of antigen (146 S) virus mouth, adjuvant (gel Goa and Saponin), received data of which it follows that the maximum level of humoral immunity is achieved with substantially smaller concentrations of these components.

The indices of the local immunity index have steadily increased with the increase of antigen concentration and Adjuvant in the vaccine. The increase in the amount of specific antigen and adjuvants in vaccines during vaccination and revaccination of animals increases the index of local immunity index, which characterizes the decrease in both the sensitivity of cells in vivo and their reproductive activity in vitro. All this testifies that the cells of the body not only have the ability to breathe and feed, but also possess specific immunity. The more accelerated dynamics of accumulation in the vaccinated animals of the level of specific 
antibodies with an increase in the concentration in the vaccine dose of vaccines of a specific antigen and adjuvant can be explained by increasing the immuno competent cells involved in the process of immuno genesis under the influence of transmitting information on the antigen through the DNA of these cells. To create specific protective properties in the cells of the body, much more specific antigen and adjuvant in the vaccine is required, and the same can be achieved with booster animals.
(C) (P) This work is licensed under Creative

To Submit Your Article Click Here: Submit Article

DOI: $10.32474 /$ CIACR.2018.01.000113

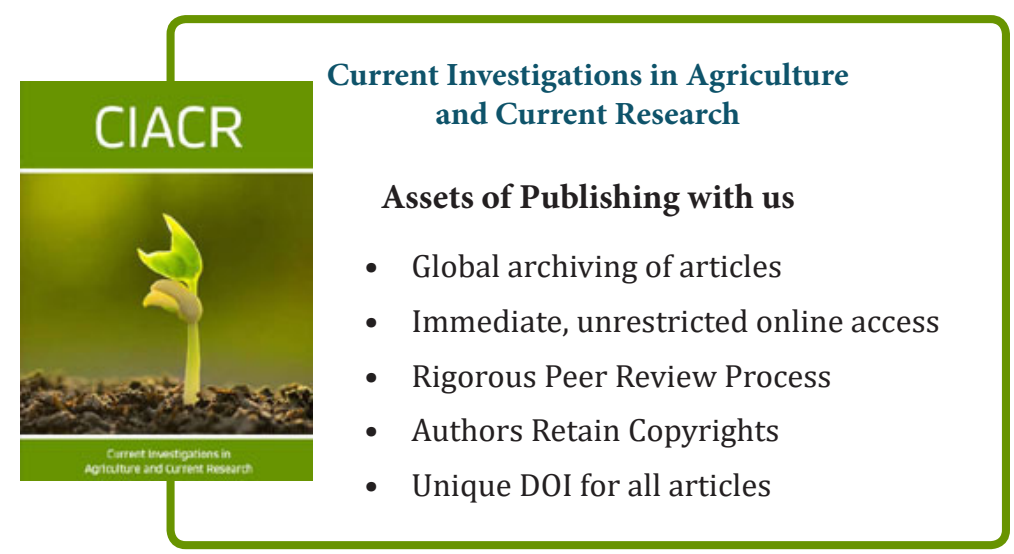

